ARTÍCULO ORIGINAL

\title{
Generación de una colonia de ratones transgénicos homocigotas K18-hACE2 para la evaluación de candidatos vacunales y terapéuticos contra el SARS-CoV-2
}

\author{
Berengeno $\mathrm{AL}^{1,2^{*}}$, Matiller $\mathrm{V}^{1,2}$, Díaz $P U^{1,2}$, Rebelindo $\mathrm{E}^{1,2}$, Rodríguez $\mathrm{FM}^{1,2}, A m w e g \mathrm{AN}^{1,2}$, Silvestrini \\ $\mathrm{P}^{1,2}$, Cattaneo $\mathrm{ML}^{1,2}$, Peralta $\mathrm{MB}^{1,2}$, Durante $\mathrm{LI}^{1,2}$, Notaro $\mathrm{US}^{1,2}$, Cainelli $\mathrm{S}^{1,2}$, Taborda $\mathrm{P}^{1,2}$, Stalder \\ $\mathrm{V}^{1,2}$, Etchevers $\mathrm{L}^{1,2}$, Rey $\mathrm{F}^{1,2}$, Salvetti $\mathrm{NR}^{1,2}$, Ortega $\mathrm{HH}^{1,2}$
}

1 Centro de Medicina Comparada (CMC), Instituto de Ciencias Veterinarias del Litoral (ICiVet Litoral), UNL / Consejo Nacional de Investigaciones Científicas y Técnicas (CONICET). Esperanza, Santa Fe, Argentina.

2 Laboratorio de Biología Celular y Molecular Aplicada, Instituto de Ciencias Veterinarias del Litoral (ICiVet-Litoral), Universidad Nacional del Litoral (UNL) / Consejo Nacional de Investigaciones Cientificas y Tecnológicas (CONICET). Esperanza, Santa Fe, Argentina.

* Correspondencia: Andrea L. Berengeno. Centro de Medicina Comparada, R.P. Kreder 2805. CP: 3080. Esperanza, Santa Fe, Argentina. E-mail: andreaberengeno@gmail.com

\# Ambos autores contribuyeron en partes iguales al desarrollo del trabajo.

RESUMEN. EL SARS-CoV-2 es el agente etiológico responsable de la enfermedad COVID-19 que inició una pandemia desde finales de 2019. Existen distintos modelos animales para esta enfermedad siendo el modelo de ratón transgénico K18-hACE2, originalmente desarrollado para el estudio del SARS-CoV-1, de gran relevancia en el contexto actual. Considerando que los ratones se comercializan como hemicigotas y dada la ausencia de ratones homocigotas para el transgén K18-hACE2, el objetivo de este trabajo fue generar una colonia de ratones homocigotas K18-hACE2- $T g / T g$ bajo condiciones ambientales controladas. Para la F1 se utilizaron 10 ratones hemicigotas (7 hembras y 3 machos), de 6-8 semanas de edad, provenientes de The Jackson Laboratory (USA). De la F1 se identificaron mediante genotipificación por PCR 78 animales: 62 hemicigotas $(T g / 0)$ y 16 salvajes $(0 / 0)$. Aquellos animales identificados como transgénicos se cruzaron con ratones C57BL/6J para seleccionar los parentales homocigotas que generaron $100 \%$ de hemicigotas en la F2. De 224 crías analizadas, se obtuvieron 7 parentales homocigotas K18-hACE2- $\mathrm{Tg} / \mathrm{Tg}$ que fueron utilizados para la colonia fundación de ratones homocigotas K18-hACE2- $T g / T g$. El modelo de ratón homocigota K18-hACE2- $T g / T g$ desarrollado en este trabajo podrá ser empleado para el estudio de la patogénesis de la enfermedad y la evaluación de posibles terapéuticos contra el SARS-CoV-2.

RESUMEN. Generation of a colony of homozygous K18-hACE2 transgenic mice for the evaluation of vaccine and therapeutic candidates against SARS-CoV-2. SARS-CoV-2 is the causative etiological agent of the COVID-19 disease that started a pandemic in late 2019. Among different animal models for this disease, the K8-hACE2 transgenic mouse model, originally developed for the study of SARS-CoV-1, proved to be of great relevance in the current context. Considering that those mice are marketed as hemizygous and given that homozygous mice for the K18-hACE2 transgene are not commercially available, this work aimed to describe the creation of a colony of homozygous K18-hACE2- $\mathrm{Tg} / \mathrm{Tg}$ mice under controlled environmental conditions. For the F1 progeny 10 hemizygous mice (7 females and 3 males), between 6-8 weeks of age, from The Jackson Laboratory (USA) were used. From the F1, 78 animals were identified by PCR genotyping: 62 hemizygous $(\mathrm{Tg} / \mathrm{O})$ and 16 wild types $(0 / 0)$. Those animals identified as transgenic were crossed with C57BL/6J mice to select the homozygous parents that generated 100\% hemizygous in F2. From a total of 224 analyzed offspring, 7 homozygous K18-hACE2- $T g / T g$ parents were obtained, which were used for the foundation colony of homozygous K18-hACE2- $T g / T g$ mice. The homozygous K18-hACE2- $\mathrm{Tg} / \mathrm{Tg}$ mouse colony developed in this work will be able to use for the study of pathogenesis and the evaluation of possible therapeutics against SARS-CoV-2. 


\section{Introducción}

En 2019, una serie de casos de neumonía severa en la ciudad de Wuhan (China) asociados a un nuevo coronavirus condujeron a un estado de alerta epidemiológico que, a tres meses de su inicio, fue declarado como pandemia por la Organización Mundial de la Salud (OMS) (Zheng, 2020; Zhou et al., 2020).

Inicialmente, este virus se denominó nuevo coronavirus 2019 (2019-nCoV, del inglés 2019-novel coronavirus) y la enfermedad originada por dicho agente etiológico fue definida como COVID-19 (del inglés coronavirus disease 2019) (Zhu et al., 2020). Posteriormente, en febrero del año 2020, la OMS junto al Comité Internacional sobre Taxonomía de Virus establecieron el nombre de coronavirus del síndrome respiratorio agudo severo 2 (SARS-CoV-2, del inglés severe acute respiratory syndrome coronavirus-2) (Gorbalenya et al., 2020; Wu F et al., 2020).

Los coronavirus (CoVs) son virus envueltos cuyo genoma consiste en una única molécula de ARN simple cadena y polaridad positiva. Pertenecen a la familia Coronaviridae (Orden Nidoviridae) y son capaces de producir signos a nivel respiratorio, gastrointestinal, hepático y neurológico en diferentes especies de aves y mamíferos, incluyendo camélidos, murciélagos, civetas, ratas, ratones, perros y gatos (Cui et al., 2019; Weiss y Leibowitz, 2011). La familia Coronaviridae se divide en dos subfamilias: Coronavirinae y Torovirinae. Los géneros alfacoronavirus y betacoronavirus de la subfamilia Coronavirinae pueden afectar a los seres humanos produciendo múltiples afecciones que incluyen desde una infección respiratoria leve hasta enfermedades más graves como el síndrome respiratorio de Medio Oriente (MERS) y el síndrome respiratorio agudo severo (SARS) (Su et al., 2016). El surgimiento de estos CoVs altamente patógenos, SARS-CoV en 2002 y MERS-CoV en 2012, generó brotes de neumonía graves evidenciando el impacto de dichas enfermedades que adquirieron dimensiones de epidemia (Lee et al., 2003; Zaki et al., 2012).

EI SARS-CoV-2 puede causar lesiones que desencadenan enfermedades respiratorias, gastrointestinales, cardiovasculares, oculares, hepáticas y neurológicas que conllevan, en algunos casos, a la muerte de pacientes afectados por COVID-19 (Hong et al., 2020; Jin et al., 2020; Prasad y Prasad, 2020).

La secuenciación genómica completa y el análisis filogenético de muestras provenientes de pacientes infectados permitieron identificar al SARS-CoV-2 como un virus del género betacoronavirus (subfamilia Coronavirinae), compartiendo el mismo género que los CoVs causantes del SARS y MERS. La similitud genómica del SARS-CoV-2 respecto del SARS-CoV (nombrado retrospectivamente como SARS-CoV-1) y MERS-CoV fue del $79 \%$ y $50 \%$, respectivamente (Lu et al., 2020; Ren et al., 2020). Además, el SARS-CoV-2 presentó una similitud genómica del $96 \%$ con el CoV de murciélago, considerado reservorio natural de los CoVs (Zhou et al., 2020).

Al igual que el resto de los CoVs, la estructura del SARSCoV-2 consta de un genoma viral que codifica proteínas estructurales y no estructurales. Las proteínas estructurales, nucleocápside $(N)$, membrana $(M)$, envoltura $(E)$, y la espícula o espiga ( $S$, del inglés Spike) se distribuyen en la cápside y la envoltura siendo responsables de la infectividad y replicación viral (Malik, 2020; Walls et al., 2020). La proteína trimérica $S$ se organiza mediante proyecciones en forma de espículas que caracterizan la estructura de la superficie del virión otorgando un aspecto de corona y de ahí el nombre de coronavirus. La proteína S del SARS-CoV-1 y SARS-CoV-2 comparten el $76,5 \%$ de su secuencia aminoacídica y presentan un alto grado de homología (Li et al., 2005; Xu et al., 2020).

El paso fundamental para el ingreso del virus en las células hospedadoras es la unión a receptores específicos ubicados en la superficie celular que interaccionen con la proteína S (Wrapp et al., 2020; Zhang et al., 2020). Dicha proteína posee dos subunidades funcionales S1 y S2, la subunidad S1 está especializada en el reconocimiento y unión con el receptor de la célula hospedadora; mientras que la función de la subunidad S2 es la fusión del virus con las membranas celulares (Hulswit et al., 2016).

El receptor para la proteína S del SARS-CoV-1 y SARSCoV-2 es la enzima convertidora de angiotensina 2 (ACE2, del inglés Angiotensin Converting Enzyme 2). La unión de la subunidad S1 al receptor ACE2 genera un complejo que es procesado catalíticamente por una serina proteasa transmembrana 2 (TMPRSS2), lo cual conduce a la escisión del ACE2 y la activación de la subunidad S2. Este proceso lleva a la fusión de la membrana viral por endocitosis que posibilita la entrada del virus y posterior liberación de su ARN en el citoplasma de la célula hospedadora (Li et al., 2003; Lu et al., 2020; Walls et al., 2020; Wan et al., 2020).

Se ha demostrado por estudios predictivos que la proteína $S$ del SARS-CoV-2 presenta una mayor afinidad por el ACE2 humano respecto del SARS-CoV-1, lo cual potencia la habilidad del SARS-CoV-2 para incrementar la tasa de contagio entre personas (Nguyen et al., 2020; Wrapp et al., 2020).

La expresión diferencial del ACE2 en los tejidos del cuerpo humano podría actuar como un potencial objetivo viral indicando la posible ruta de la infección por SARS-CoV-2. Se ha identificado la presencia del ACE2 en la superficie extracelular de distintos órganos como cerebro, corazón, riñones, pulmones e intestino y específicamente, en las células epiteliales alveolares tipo II de pulmón y en enterocitos del intestino delgado (Harmer et al., 2002; Hamming et al., 2004). La mayor expresión del ACE2 en estos tejidos se correlaciona con los síntomas principales de la enfermedad por COVID19 , en tre los que se incluyen fiebre, tos seca, mialgia, 
fatiga y dificultad respiratoria $y$, en menor grado, diarrea, náuseas y vómitos (Huang et al., 2020).

Dada la emergencia debida a la falta de inmunidad preexistente frente al SARS-CoV-2, numerosos trabajos científicos se encuentran dirigidos al desarrollo de posibles alternativas terapéuticas a corto y largo plazo para el tratamiento y la prevención de esta enfermedad que reduzca la morbilidad y mortalidad de los pacientes infectados.

Si bien los modelos in vitro basados en cultivos de células, tejidos $u$ organoides posibilitan el estudio de la biología del SARS-CoV-2 y el abordaje de blancos celulares bajo condiciones controladas de laboratorio, reflejan parcialmente la complejidad estructural e interrelación funcional de los sistemas de órganos de un ser humano (Hui et al., 2020; Xia et al., 2020).

A diferencia de los modelos in vitro, los modelos in vivo brindan una herramienta valiosa para el análisis de la historia natural de la enfermedad y son de gran relevancia para la evaluación de la seguridad y eficacia de posibles antivirales y vacunas contra el SARS-CoV-2 (Yuan et al., 2020). Distintos estudios han reportado que tanto pequeños como grandes animales, entre ellos ratones, hámsters, conejos, murciélagos, gatos, perros, hurones y primates no humanos, pueden infectarse con el SARS-CoV-2, lo cual posibilitaría su empleo como modelos in vivo del COVID-19 (Muñoz-Fontela et al., 2020; Rosa et al., 2021).

Los ratones presentan algunas características propias que los han posicionado como modelos in vivo de excelencia dentro de las que se encuentran el background genético conocido, tamaño, disponibilidad, facilidad de manipulación y alojamiento, bajo costo y su utilización previa en el estudio de la patogénesis de los CoVs humanos, que les otorga cierta ventaja frente al resto de los animales (Cockrell et al., 2018).

El SARS-CoV-2, al igual que el SARS-CoV-1, presenta una mayor afinidad por el receptor de la enzima convertidora de angiotensina I humana peptidil dipeptidasa A 2 (hACE2) respecto de otras especies animales (Li et al., 2006; Wan et al., 2020). Li et al., (2004) han demostrado que la replicación del SARS-CoV-1 en ratones salvajes no era efectiva, debido a que el receptor del ratón (mACE2) se une menos eficientemente que el hACE2 a la subunidad S1 de la proteína $\mathrm{S}$ del SARS-CoV-1. Dicho fenómeno asociado a la replicación viral, también se observó en animales infectados con el SARS-CoV-2 debido a la falta de interacciones eficientes entre la proteína $S$ y el mACE2 (Zhou et al., 2020). Como consecuencia de esta limitación, se han desarrollado modelos de ratones transgénicos que expresan la proteína hACE2 para aumentar la replicación viral y reproducir la signología ligada al SARS (McCray et al., 2007; Tseng et al., 2007; Bao et al., 2020; Jiang et al., 2020).
En los modelos de ratones transgénicos descriptos hasta el momento, la expresión de la hACE2 se encuentra asociada al control de promotores como la queratina 18 humana (K18) (McCray et al., 2007); un promotor CAG compuesto por la secuencia potenciadora de citomegalovirus, el promotor del gen de la $\beta$ actina de pollo y el sitio de poliadenilación y splicing del gen de la globina de conejo (Tseng et al., 2007); la secuencia codificante del hACE2 (Bao et al., 2020) o el factor nuclear homólogo-4 del hepatocito (HFH4) (Jiang et al., 2020). Se han reportado distintos niveles de replicación viral en los órganos blanco y diferencias en la patogénesis de la enfermedad, producto de la variabilidad en la expresión celular y tisular del hACE2 en estos modelos transgénicos. La letalidad de los animales se ha vinculado a fallas multiorgánicas, incluyendo el daño observado en los pulmones, considerado el principal órgano blanco del SARS-CoV-2 en pacientes que sufren COVID-19 (Wu F et al., 2020).

Particularmente el modelo de ratón transgénico K18hACE2, originalmente desarrollado por McCray et al., (2007) para el estudio del SARS-CoV-1, adquiere gran importancia desde la emergencia del SARS-CoV-2.

Los rasgos característicos de este modelo se encuentran estrechamente relacionados con la inserción del transgén k18-hACE2. El diseño del transgén de $6,8 \mathrm{~kb}$ consistió en una secuencia genómica de $2,5 \mathrm{~kb}$ del gen de la queratina 18 humana (KRT18) con el promotor, el primer intrón que presentó una mutación en el sitio aceptor de empalme 3' para reducir el salto del exón y una secuencia potenciadora de la traducción (TE) del virus del mosaico de la alfalfa corriente arriba de la secuencia codificante de la hACE2 seguida de los exones 6-7 y la señal poli (A) del gen K18 humano. Estos elementos posibilitaron un mayor nivel de expresión y especificidad epitelial.

En los ratones transgénicos K18-hACE2 infectados con SARS-CoV-1, la expresión del ARNm del hACE2 se detectó en pulmones, hígado, riñones, colon y cerebro. Los animales resultaron altamente susceptibles a la infección evidenciada por una alta carga viral e inflamación en pulmones, pérdida de peso significativa y morbilidad a partir del cuarto día pos-infección (McCray et al., 2007).

Basado en los antecedentes previamente mencionados sobre la mayor afinidad del SARS-CoV-2 por el hACE2 comparado al SARS-CoV-1 junto a la susceptibilidad de los animales transgénicos K18-hACE2 frente al SARSCoV-1, se demuestra que este modelo animal también puede ser considerado para el estudio del SARS-CoV- 2 .

Se ha reportado que la infección por SARS-CoV-2 en ratones K18-hACE2 produjo daño pulmonar agudo fatal, similar al SARS-CoV-1, asociado con niveles altos de citoquinas inflamatorias y acumulación de células inmune en los pulmones (McCray et al., 2007; Moreau et al., 2020; Winkler et al., 2020; Zheng et al., 2021). En algunos trabajos, el SARS-CoV-2 generó infecciones 
neuronales letales en ratones K18-hACE2 (Carossino et al., 2021; Kumari et al., 2021). Oladunni et al., (2020) demostraron una respuesta inflamatoria del tipo "tormenta de citoquinas" en ratones K18-hACE2 infectados con SARS-CoV-2 similar a la observada en seres humanos. Además, Zheng et al., (2021) comprobaron que el pretratamiento con plasma de pacientes recuperados de COVID-19 en ratones K18hACE2 infectados previno los síntomas de neumonía grave.

En consecuencia, dado que los ratones K18-hACE2 infectados con el SARS-CoV-2 presentan muchos de los signos clínicos observados en pacientes con COVID-19 puede considerarse que constituyen un modelo preclínico apropiado para el estudio de la patogénesis de esta enfermedad y la evaluación de posibles candidatos terapéuticos y preventivos contra la infección por el SARS-CoV-2.

Si bien en la actualidad existen diversos modelos de ratones para el transgén K18-hACE2, el modelo propuesto en este trabajo se basa en el ratón transgénico B6.Cg-Tg(K18-ACE2)2Prlmn/J producido por The Jackson Laboratory. En dicho modelo, el constructo conteniendo el transgén K18-hACE2 ha sido generado en embriones (C57BL/6J $\times$ SJL/J), produciendo ratones transgénicos K18-hACE2 con 8 copias completas del transgén los cuales fueron retrocruzados con ratones C57BL/6J para el mantenimiento y ampliación de la colonia. Sin embargo, estos animales son provistos como hemicigotas para el transgén K18-hACE2 y por ello, como producto de su apareamiento, pueden transmitir un número variable de copias a su descendencia dependiendo de aquellas copias disponibles en los respectivos progenitores. Aunque existen distintos métodos para diferenciar entre ratones hemicigotas y homocigotas, los cebadores utilizados en este trabajo están diseñados para detectar la construcción del transgén K18-hACE2 pero no permiten distinguir entre ambos genotipos. Consecuentemente, esto conlleva la necesidad de genotipificar por técnicas de PCR a todos los animales para identificar los portadores, ya que no existen variaciones fenotípicas detectables que permitan hacerlo.

Por ello, el objetivo de este trabajo fue la generación de una colonia de ratones transgénicos homocigotas a partir de los ratones B6.Cg- $\mathrm{Tg}(\mathrm{K} 18-\mathrm{ACE} 2) 2 \mathrm{PrImn} / \mathrm{J}$ (K18hACE2). Esto facilitaría la producción de descendencia portadora del gen de interés, sin la necesidad de realizar el genotipado por PCR. Además, reduciría el tiempo entre la producción y uso de los animales, espacio de alojamiento, costos y fundamentalmente, permitirá disponer de un stock permanente de ratones homocigotas K18-hACE2 que puedan ser utilizados para la ejecución de ensayos preclínicos de posibles fármacos para el tratamiento y prevención de COVID-19.

\section{Materiales y métodos}

\section{Animales}

Se adquirieron desde Estados Unidos 10 ratones hemicigotas ( 7 hembras y 3 machos, de una edad estimada de 6-8 semanas) para el transgén K18-ACE2 (Número de stock 034860, B6.Cg-Tg(K18-ACE2) 2Prlmn/J, The Jackson Laboratory, Bar Harbor, ME, USA).

Los animales fueron alojados en instalaciones del Centro de Medicina Comparada (CMC, Instituto de Ciencias Veterinarias del Litoral, UNL-CONICET) habilitadas y certificadas por ANMAT y SENASA, con fotoperíodo de $12 \mathrm{~h}$ luz $/ 12 \mathrm{~h}$ oscuridad, temperatura controlada de entre 20 y $24{ }^{\circ} \mathrm{C}$, con libre acceso a alimento comercial (GEPSA, Argentina) y agua bajo condiciones ambientales controladas (sistema acondicionador de aire con filtrado de alta eficiencia, racks de cajas ventiladas individualmente (IVC), estaciones de recambio y cabinas de seguridad biológica con protección de clase II (Allentown Inc., USA) y sistema de monitoreo automático de condiciones ambientales). Los animales del CMC están definidos microbiológicamente como libres de patógenos especificados y son sometidos a controles sanitarios internos $y$ externos, realizados en el Centro de Diagnóstico Veterinario (Buenos Aires, Argentina) y Charles River Laboratories (USA), donde se evalúan los microrganismos incorporados en el listado de FELASA (Federation of European Laboratory Animal Science Associations FELASA, Mähler et al., 2014). Los ratones K18-ACE2 fueron controlados sanitariamente en el momento del ingreso de manera permanente por la unidad de monitoreo ambiental y sanitario del CMC, de acuerdo con los procedimientos operativos estandarizados vigentes. Previo al inicio de las actividades, los animales fueron sometidos a una cuarentena de dos semanas.

\section{Generación de la F1 hemicigota}

A partir de los ratones hemicigotas K18-hACE2 adquiridos, se seleccionaron progenitores machos y hembras para el armado de distintos planteles reproductores del tipo K18-hACE2 $\mathrm{Tg} / 0 \times \mathrm{K} 18-\mathrm{hACE2} \mathrm{Tg} / 0$ designados como colonia fundadora filial 0 (FO). Para ello, se utilizó un sistema de apareamiento en harem mediante parejas formadas por un macho K18-hACE2 con dos hembras K18-hACE2. Las crías producto de estos apareamientos, denominadas como F1, fueron genotipificadas para determinar la presencia del transgén en la descendencia como se detallará más adelante (Figura 1).

\section{Selección de la F1 homocigota}

Como producto de la genotipificación se obtuvieron los animales F1, portadores del gen hACE2, tanto homo como hemicigotas. Para lograr la identificación de los animales homocigotas, se recurrió a aparear cada ratón 
transgénico con un animal $\mathrm{C} 57 \mathrm{BL} / 6 \mathrm{~J}$ no portador del transgen (wild type) de la colonia disponible en el CMC y analizar la segregación de los diferentes genotipos en las crías generadas en el primer apareamiento. Se consideraron homocigotas y se reservaron para la posterior generación de la colonia homocigota, aquellos animales transgénicos donde el análisis de la descendencia de la pareja generó un $100 \%$ de las crías positivas en la genotipificación del transgén en las camadas generadas.

\section{Generación de la F2 homocigota}

Una vez identificados los progenitores homocigotas K18-hACE2 de la F1, se comenzó con el cruzamiento de los individuos homocigotas mediante un sistema por parejas monogámicas (una hembra homocigota con un macho homocigota). Las crías obtenidas del cruce de parejas homocigotas K18-hACE2- $T g / T g$ se genotipificaron para determinar la presencia del gen hACE2 (Figura 1).

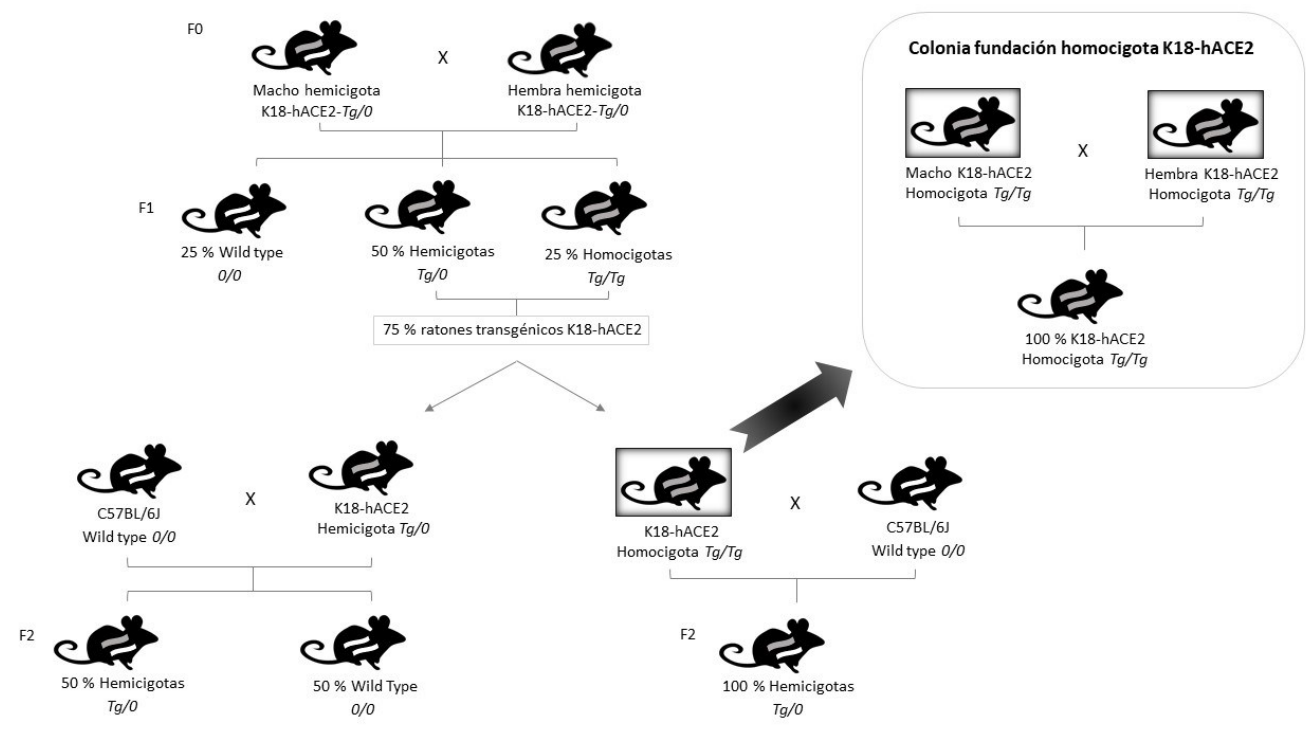

Figura 1. Generación de una línea de ratones homocigotas para el transgén K18-hACE2 a partir de ratones hemicigotas K18-hACE2 y C57BL/6J. La F1, generada mediante el cruce de ratones hemicigotas K18-hACE2 provenientes de The Jackson Laboratory produjo $75 \%$ de animales transgénicos. El análisis de la descendencia de la F2, producto del cruce de dichos animales transgénicos con ratones C57BL/6J wild type 0/0 permitió identificar a los progenitores, siendo homocigota para el gen de interés en aquel caso donde el $100 \%$ de las crías resultaron hemicigotas K18-hACE2. Los parentales (macho y hembra) homocigotas identificados se cruzaron produciendo una descendencia con la totalidad de las crías portadoras del transgén K18-hACE2.

\section{Extracción de ADN genómico}

Para la extracción del ADN se empleó el protocolo HotSHOT para la preparación de ADNg de ratón de calidad PCR descripto por Truett et al., (2000). A un fragmento de tejido extraído del extremo distal de la cola (aproximadamente 1-3 $\mathrm{mm}$ ) de animales jóvenes de 10-21 días se le adicionó $75 \mu \mathrm{l}$ de buffer de lisis alcalina ( $\mathrm{NaOH} 25 \mathrm{mM}$, EDTA $0,2 \mathrm{mM}, \mathrm{pH}$ 12). En un termociclador (Techne TC-312, UK), se realizó la lisis durante $1 \mathrm{~h}$ a $98^{\circ} \mathrm{C}$ seguido por $5 \mathrm{~min}$ a $15^{\circ} \mathrm{C}$ y se dejó enfriar a $4-8{ }^{\circ} \mathrm{C}$ para luego continuar el procesamiento de la muestra. Posteriormente, se adicionaron $75 \mu \mathrm{l}$ de buffer de neutralización (Tris-HCl $40 \mathrm{mM}, \mathrm{pH} 5,5$ ) y se centrifugó a $2000 \mathrm{~g}$ durante $3 \mathrm{~min}$. Una alícuota del ADNg obtenido fue utilizada para la realización de la PCR.

PCR en tiempo real para la detección del transgén K18hACE2

Tres oligonucleótidos diseñados por The Jackson Laboratory (53437, 53438 y 53439) se emplearon en dos reacciones de PCR (reacciones A y B) para la identificación de los animales portadores del transgén K18-hACE2 (Tabla 1). En un volumen final de $20 \mu \mathrm{l}$, se agregaron $2 \mu \mathrm{l}$ del ADNg obtenido en una mezcla de reacción conteniendo $3 \mathrm{mM} \mathrm{MgCl}_{2} ; 0,2 \mathrm{mM}$ dNTP; 0,5 $\mu \mathrm{M}$ de cebadores sentido y antisentido; 0,5 $\mu \mathrm{M}$ de SYBR GREEN y $0,08 \mathrm{U} / \mu \mathrm{l}$ de Taq polimerasa (ThermoFisher, Life Technologies, USA). Las reacciones de PCR se efectuaron en un termociclador para $P C R$ en tiempo real (StepOne, Applied Biosystems, USA) empleando las siguientes condiciones de amplificación: 1 ciclo a $95{ }^{\circ} \mathrm{C}$ por $5 \mathrm{~min} ; 10$ ciclos de $95{ }^{\circ} \mathrm{C}-20 \mathrm{~s}, 65{ }^{\circ} \mathrm{C}-20 \mathrm{~s}\left(-0,5{ }^{\circ} \mathrm{C}\right.$ disminuyendo por ciclo) y $68^{\circ} \mathrm{C}-20 \mathrm{~s} ; 28$ ciclos de $95^{\circ} \mathrm{C}$ $20 \mathrm{~s}, 60{ }^{\circ} \mathrm{C}-20$ s y $72{ }^{\circ} \mathrm{C}-20 \mathrm{~s}$ y finalmente 1 ciclo a $72{ }^{\circ} \mathrm{C}$ por $10 \mathrm{~min}$. Como controles positivos se utilizaron muestras provenientes de los ratones $\mathrm{C} 57 \mathrm{BL} / 6 \mathrm{~J}$ para la reacción $\mathrm{A}$ (bandas correspondientes a $221 \mathrm{pb}$ ) y de los ratones hemicigotas $\mathrm{K} 18$-hACE2- $\mathrm{Tg} / 0$ en la reacción $\mathrm{B}$ (bandas de $170 \mathrm{pb}$ ) (Figura 2). 


\section{Resultados}

Mediante el cruce parental F0 de ratones hemicigotas K18-hACE2, se obtuvo la primera generación de animales. En la F1 se detectaron, sobre un total de 78 animales, 62 que contenían el transgén (hemicigotas $T g / 0$ u homocigotas $T g / T g$ ) y 16 no transgénicos (0/0). Los animales transgénicos genotipificados representaron un $79 \%$ del total de los animales analizados. Dicho porcentaje se aproxima al valor esperado de segregación que es del 75 \% (Tabla 2).

La progenie de ratones transgénicos obtenidos de la F0 se apareó inicialmente con ratones C57BL/6J (sin el transgén) realizándose 23 cruces que generaron una F2 con distinta proporción de genotipos (Tablas 3 y 4). Como resultado de los cruces 1 a 16, se obtuvieron 71 hemicigotas $(\mathrm{Tg} / 0)$ y 79 ratones sin el transgén $(0 / 0)$, lo cual indica que aproximadamente la mitad de los animales tenían el transgén y la mitad restante no. De este modo, el progenitor transgénico utilizado en estos cruces presenta un genotipo hemicigota ( $\mathrm{Tg} / \mathrm{O}$ ) (Tabla 3).

En los cruces 17 a 23 , se identificaron 74 animales hemicigotas $(\mathrm{Tg} / 0)$ y ninguno sin el transgén (0/0). Así, el $100 \%$ de los animales obtenidos en la F2 como transgénicos indicó que el parental correspondiente (macho o hembra) fue portador del gen hACE2 como homocigota $(T g / T g)$ (Tabla 4).

A partir del cruce de homocigotas K18-hACE2 como parte de la producción de un núcleo de fundación de una colonia de homocigotas, el $100 \%$ de las crías fueron portadoras del gen hACE2 obteniéndose un total de 34 crías homocigotas, 10 machos y 24 hembras.

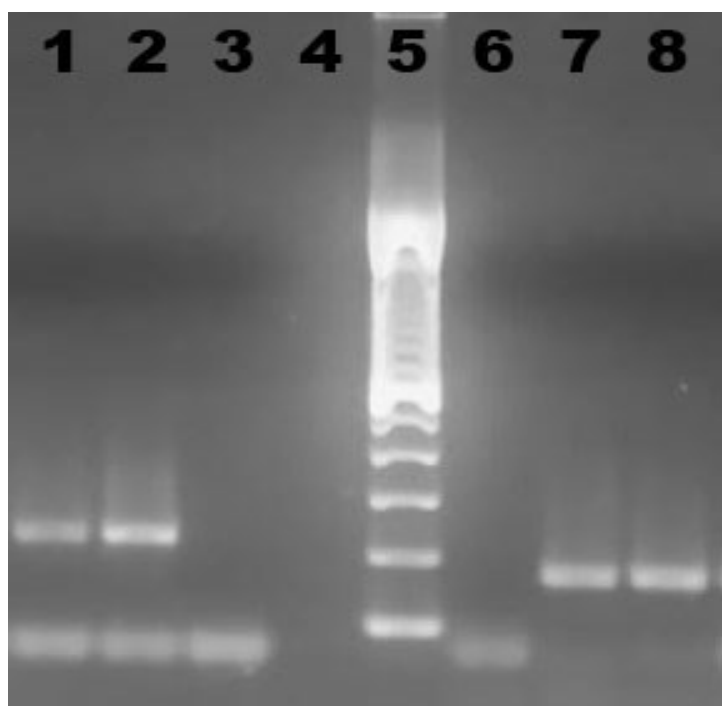

Figura 2. Imagen representativa de un gel de agarosa $2 \%$. Calles 1 y 2: bandas correspondientes a la reacción A (221pb) de los ratones $\mathrm{C} 57 \mathrm{BL} / 6 \mathrm{~J}$ usados como controles positivos. Calle 3: control negativo reacción A. Calle 4: sin siembra. Calle 5 : Estándar de ADN de 100pb. Calle 6: control negativo reacción B. Calles 7 y 8: bandas propias de la reacción B (170pb) de los ratones hemicigotas K18-hACE2-Tg/0 empleados como controles positivos.
Tabla 1. Cebadores empleados para la genotipificación de los ratones K18-hACE2.

\begin{tabular}{|c|c|c|c|c|}
\hline Nombre & Secuencia & $\begin{array}{c}\text { Reac. } \\
\text { PCR }\end{array}$ & Genotipo & Referencias \\
\hline 53437 & $\begin{array}{l}\text { 5'-GACCCCTGAGG } \\
\text { GTTTCATATAG-3' }\end{array}$ & $B$ & Mutante & $\begin{array}{c}\text { Oligo } \\
\text { Synthesis }\end{array}$ \\
\hline 53438 & $\begin{array}{l}\text { 5'-CACCAACACA } \\
\text { GTTTCCCAAC-3' }\end{array}$ & $\begin{array}{c}\text { A y } \\
B\end{array}$ & Mutante & $\begin{array}{l}\text { Resource. } \\
\text { Genotyping } \\
\text { primers for }\end{array}$ \\
\hline 53439 & $\begin{array}{c}\text { 5'-AAGTTGGAGAA } \\
\text { GATGCTGAAAGA- } \\
3^{\prime}\end{array}$ & A & Salvaje & $\begin{array}{l}\text { K18-hACE2 } \\
\text { mice. The } \\
\text { Jackson } \\
\text { Laboratory. }\end{array}$ \\
\hline
\end{tabular}

Tabla 2. Generación de la F1 hemicigota. Se indican los resultados de la progenie de los cruces F0 K18-hACE2 x K18hACE2, ambos hemicigotas $\mathrm{Tg} / 0$.

\begin{tabular}{|c|c|c|}
\hline \multicolumn{3}{|c|}{ Cruza parental Fo } \\
\hline \multirow[b]{2}{*}{ Cruza } & \multicolumn{2}{|c|}{ Genotipo de las crías } \\
\hline & $\begin{array}{c}\text { Mutante } \\
\text { (Homocigota } T g / T g \text { y } \\
\text { hemicigota } T g / 0 \text { ) } \\
\end{array}$ & Salvaje (0/0) \\
\hline $\begin{array}{l}\text { Macho hemicigota K18-hACE2- } \mathrm{Tg} / 0 \\
\mathrm{x} \\
\text { Hembra hemicigota K18-hACE2- } \mathrm{Tg} / 0\end{array}$ & 62 & 16 \\
\hline $\mathrm{N}^{\circ}$ total de crías analizadas & \multicolumn{2}{|l|}{78} \\
\hline$\%$ de crías & 79 & 21 \\
\hline
\end{tabular}

Tabla 3. Generación de la F1 hemicigota. Se indican los resultados de la progenie de los cruces F0 K18-hACE2 x K18hACE2, ambos hemicigotas $\mathrm{Tg} / 0$.

\begin{tabular}{|c|c|c|c|}
\hline \multicolumn{4}{|c|}{ Cruza parental F1 } \\
\hline \multirow{2}{*}{\multicolumn{2}{|c|}{ Tipo y cruza $\mathrm{N}^{\circ}$}} & \multicolumn{2}{|c|}{ Genotipo de las crías } \\
\hline & & \multirow{2}{*}{\begin{tabular}{|c|}
$\begin{array}{c}\text { Hemicigota } \\
(\mathrm{Tg} / \mathrm{O})\end{array}$ \\
4
\end{tabular}} & \multirow{2}{*}{\begin{tabular}{|c|}
$\begin{array}{c}\text { Salvaje } \\
(0 / 0)\end{array}$ \\
3
\end{tabular}} \\
\hline \multirow{16}{*}{$\begin{array}{c}\text { Mutante } \\
\text { (Homocigota } T g / T g \text { o hemicigota } T g / 0) \\
\text { x } \\
\text { C57BL/6J } \\
\text { Salvaje }(0 / 0)\end{array}$} & 1 & & \\
\hline & 2 & 4 & 3 \\
\hline & 3 & 4 & 3 \\
\hline & 4 & 4 & 3 \\
\hline & 5 & 2 & 3 \\
\hline & 6 & 1 & 3 \\
\hline & 7 & 5 & 3 \\
\hline & 8 & 3 & 5 \\
\hline & 9 & 5 & 8 \\
\hline & 10 & 9 & 12 \\
\hline & 11 & 4 & 7 \\
\hline & 12 & 3 & 7 \\
\hline & 13 & 2 & 3 \\
\hline & 14 & 4 & 1 \\
\hline & 15 & 4 & 11 \\
\hline & 16 & 13 & 4 \\
\hline $\mathrm{N}^{\circ}$ de crías $/ \mathrm{N}^{\circ}$ total de crías & & $71 / 150$ & $79 / 150$ \\
\hline$\%$ de crías & & 47 & 53 \\
\hline
\end{tabular}


Tabla 4. Selección de progenitores homocigotas en la F1. Se indican los resultados de la progenie de los cruces de parentales K18-hACE2 (homocigota $T g / T g$ o hemicigota $T g / 0$ ) y C57BL/6J salvaje (0/0). La numeración de los cruces es arbitraria. La proporción de los genotipos provenientes de los cruces $\mathrm{N}^{\circ} 17-21$ y 23 es indicativa de que dicho parental mutante posee genotipo homocigota $(T g / T g)$. * Si bien todos los animales genotipificados en la cruza $\mathrm{N}^{\circ} 22$ resultaron hemicigotas se dispone de un número total bajo de crías como para seleccionar dicho progenitor como homocigota.

\begin{tabular}{|c|c|c|c|}
\hline \multicolumn{4}{|c|}{ Cruza parental F1 } \\
\hline \multirow{2}{*}{\multicolumn{2}{|c|}{ Tipo y cruza $\mathrm{N}^{\circ}$}} & \multicolumn{2}{|c|}{ Genotipo de las crías } \\
\hline & & \multirow{2}{*}{\begin{tabular}{|c}
$\begin{array}{c}\text { Hemicigota } \\
(\mathrm{Tg} / \mathrm{O})\end{array}$ \\
5
\end{tabular}} & \multirow{2}{*}{\begin{tabular}{|c|}
$\begin{array}{c}\text { Salvaje } \\
(0 / 0)\end{array}$ \\
0 \\
\end{tabular}} \\
\hline \multirow{7}{*}{$\begin{array}{c}\text { Mutante } \\
\text { (Homocigota } T g / T g \text { o hemicigota } T g / 0) \\
\text { x } \\
\text { C57BL/6J } \\
\text { Salvaje }(0 / 0)\end{array}$} & 17 & & \\
\hline & 18 & 11 & 0 \\
\hline & 19 & 17 & 0 \\
\hline & 20 & 15 & 0 \\
\hline & 21 & 7 & 0 \\
\hline & 22 & $4^{*}$ & 0 \\
\hline & 23 & 15 & 0 \\
\hline $\mathrm{N}^{\circ}$ de crías $/ \mathrm{N}^{\circ}$ total de crías & & $74 / 74$ & $0 / 74$ \\
\hline$\%$ de crías & & 100 & 0 \\
\hline
\end{tabular}

\section{Discusión}

Desde hace mucho tiempo, los modelos animales han resultado esenciales para la validación preclínica de posibles fármacos y vacunas previo a las fases clínicas en seres humanos. La pandemia causada por el SARSCoV-2 ha llevado a la necesidad de contar con modelos animales apropiados para el estudio de la COVID-19.

En nuestro laboratorio a partir de ratones hemicigotas K18-hACE2- $\mathrm{Tg} / 0$, logramos generar una colonia de ratones homocigotas K18-hACE2- Tg/Tg controlados mediante la genotipificación por PCR de todos los animales obtenidos.

La detección de los animales homocigotas para la generación de una colonia que no requiera la continua genotipificación de los animales resulta fundamental para acortar los tiempos en la obtención de animales y abaratar los costos en estudios preclínicos de eficacia.

Si bien se pueden emplear ratones no transgénicos para algunos estudios iniciales como la evaluación de la respuesta inmune humoral o el análisis de posibles vías de administración de un candidato terapéutico, al no desarrollar signos propios de la enfermedad, dichos animales tienen una utilidad limitada. Esto se debe a la capacidad diferencial de unión del receptor murino mACE2 al SARS-CoV-2 que impide que estos animales puedan ser infectados eficientemente $y$, por ende, no resulta factible su utilización como modelo traslacional contra la infección por el SARS-CoV-2 (Muñoz-Fontela et al., 2020).

En los estudios de eficacia de los candidatos vacunales y terapéuticos es de vital importancia el desarrollo de modelos in vivo que repliquen lo mejor posible los aspectos de la patogenia observados en la enfermedad que se presenta en humanos para poder obtener datos fidedignos antes del inicio de los estudios clínicos (Moreau et al., 2020; Oladunni et al., 2020; Jian Zheng et al., 2021).

Es de destacar que los ratones transgénicos K18-hACE2 son susceptibles a la infección por SARS-CoV-2 tanto experimental como naturalmente. Es por ello que estos animales transgénicos deben ser mantenidos en condiciones ambientales altamente controladas y su manejo debe estar a cargo de personas entrenadas $y$ con las medidas de seguridad primarias y barreras secundarias que impidan la infección natural. El modelo animal que se desarrolla mediante la infección experimental requiere condiciones de seguridad biológica 3 (BSL3) de acuerdo con la recomendación de la OMS (2021). En este sentido, las instalaciones requeridas para el desarrollo de los modelos de infección no están disponibles en los laboratorios de investigación habituales, sino que requieren diseño, capacitación del personal, equipamiento de seguridad especial y procedimientos apropiados. Los costos asociados al uso de un laboratorio BSL3 sumados a los relacionados con la producción de ratones transgénicos para el desarrollo de los modelos de infección son elevados. La generación y mantenimiento de una colonia de ratones transgénicos homocigotas permitiría un desarrollo eficaz de los modelos de infección y disminuiría los costos relacionados con la genotipificación continua de los animales a ser usados en estos estudios.

En conclusión, la estrategia efectiva presentada en este trabajo ha permitido desarrollar una colonia estable de ratones homocigotas K18-hACE2- $T g / T g$ que podrán ser utilizados para la evaluación preclínica de candidatos vacunales o antivirales en pos de frenar el avance de la pandemia del COVID-19.

\section{Referencias}

Bao L, Deng W, Huang B, Gao H, Liu J, Ren L, Wei Q, Yu P, Xu Y, Qi F, Qu Y, Li F, L Q, Wang W, Xue J, Gong S, Liu M, Wang G, Wang S, Qin C. 2020. The pathogenicity of SARS-CoV-2 in hACE2 transgenic mice. Nature 583: 830-833.

Carossino M, Montanaro P, O'Connell A, Kenney D, Gertje H, Grosz KA, Kurnick SA, Bosmann M, Saeed M, Balasuriya UB, Douam F,Crossland NA. 2021. Fatal neuroinvasion of SARSCoV-2 in K18-hACE2 mice is partially dependent on hACE2 expression. BioRxiv. doi:10.1101/2021.01.13.425144.

Cockrell AS, Leist SR, Douglas MG, Baric RS. 2018. Modeling pathogenesis of emergent and pre-emergent human coronaviruses in mice. Mammalian Genome 29: 367-383.

Cui J, Li F, Shi ZL. 2019. Origin and evolution of pathogenic coronaviruses. Nat. Rev. Microbiol. 17: 181-192.

Gorbalenya AE, Baker SC, Baric RS, de Groot RJ, Drosten C, Gulyaeva AA, Haagmans BL, Lauber C, Leontovich AM, Neuman BW, Penzar D, Perlman S, Poon LLM, Samborskiy DV, Sidorov 
IA, Sola I, Ziebuhr J. 2020. The species Severe acute respiratory syndrome-related coronavirus: classifying 2019-nCoV and naming it SARS-CoV-2. Nat. Microbiol. 5: 536-544.

Hamming I, Timens W, Bulthuis MLC, Lely AT, Navis GJ, van Goor H. 2004. Tissue distribution of ACE2 protein, the functional receptor for SARS coronavirus. A first step in understanding SARS pathogenesis. J. Pathol. 203: 631-637.

Harmer D, Gilbert M, Borman R, Clark KL. 2002. Quantitative mRNA expression profiling of ACE 2, a novel homologue of angiotensin converting enzyme. FEBS Lett. 532: 107-110.

Hong N, Yu W, Xia J, Shen Y, Yap M, Han W. 2020. Evaluation of ocular symptoms and tropism of SARS-CoV-2 in patients confirmed with COVID-19. Acta. Ophthalmol. 98: e649-e655.

Huang $C$, Wang $Y$, Li X, Ren L, Zhao J, Hu Y, Zhang L, Fan G, Xu J, Gu X, Cheng Z, Yu T, Xia J, Wei Y, Wu W, Xie X, Yin W, Li H, Liu M, Cao B. 2020. Clinical features of patients infected with 2019 novel coronavirus in Wuhan, China. Lancet 395: 497-506.

Hui KPY, Cheung MC, Perera RAPM, Ng KC, Bui CHT, Ho JCW, Ng MMT, Kuok DIT, Shih KC, Tsao SW, Poon LLM, Peiris M, Nicholls JM, Chan MCW. 2020. Tropism, replication competence, and innate immune responses of the coronavirus SARS-CoV-2 in human respiratory tract and conjunctiva: an analysis in ex-vivo and in-vitro cultures. Lancet. Resp. Med. 8: 687-695.

Hulswit RJ, de Haan CAM, Bosch B. 2016. Coronavirus Spike Protein and Tropism Changes. In Adv. Virus. Res. Elservier Inc. Pp. 29-57. ISSN 0065-3527.

Jiang RD, Liu MQ, Chen $Y$, Shan C, Zhou YW, Shen XR, Li Q, Zhang L, Zhu Y, Si HR, Wang Q, Min J, Wang X, Zhang W, Li B, Zhang HJ, Baric RS, Zhou P, Yang XL, Shi ZL. 2020. Pathogenesis of SARS-CoV-2 in Transgenic Mice Expressing Human Angiotensin-Converting Enzyme 2. Cell 182: 50-58.

Jin X, Lian JS, Hu JH, Gao J, Zheng L, Zhang YM, Hao SR, Jia HY, Cai H, Zhang XL, Yu GD, Xu KJ, Wang XY, Gu JQ, Zhang SY, Ye CY, Jin CL, Lu YF, Yu X, Yang, Y. (2020). Epidemiological, clinical and virological characteristics of 74 cases of coronavirusinfected disease 2019 (COVID-19) with gastrointestinal symptoms. Gut 69: 1002-1009.

Kumari P, Rothan HA, Natekar JP, Stone S, Pathak H, Strate PG Arora K, Brinton MA, Kumar M. 2021. Neuroinvasion and encephalitis following intranasal inoculation of SARS-CoV-2 in K18-hACE2 mice. Viruses 13: 1-12.

Lee $\mathrm{N}$, Hui $\mathrm{D}$, Wu A, Chan $\mathrm{P}$, Cameron $\mathrm{P}$, Joynt GM, Ahuja A, Yung MY, Leung CB, To KF, Lui SF, Szeto CC, Chung S, Sung JJY. 2003. A Major Outbreak of Severe Acute Respiratory Syndrome in Hong Kong. New. Engl. J. Med. 348: 1986-1994.

Li F, Li W, Farzan M, Harrison SC. 2005. Structural biology: Structure of SARS coronavirus spike receptor-binding domain complexed with receptor. Science 309: 1864-1868.

Li W, Greenough TC, Moore MJ, Vasilieva N, Somasundaran M, Sullivan JL, Farzan M, Choe H. 2004. Efficient Replication of Severe Acute Respiratory Syndrome Coronavirus in Mouse Cells Is Limited by Murine Angiotensin-Converting Enzyme 2. J. Virol. 78: 11429-11433.

Li W, Moore MJ, Vasilieva N, Su J, Wong SK, Berne MA,
Somasundaran M, Sullivan JL, Luzuriaga K, Greenough TC, Choe $\mathrm{H}$, Farzan M. 2003. Angiotensin-converting enzyme 2 is a functional receptor for the SARS coronavirus. Nature 426: 450454.

Li W, Wong SK, Li F, Kuhn JH, Huang IC, Choe H, Farzan M. 2006. Animal Origins of the Severe Acute Respiratory Syndrome Coronavirus: Insight from ACE2-S-Protein Interactions. J. Virol. 80: 4211-4219.

Lu R, Zhao X, Li J, Niu P, Yang B, Wu H, Wang W, Song H, Huang B, Zhu N, Bi Y, Ma X, Zhan F, Wang L, Hu T, Zhou H, Hu Z, Zhou W, Zhao L, Tan W. 2020. Genomic characterisation and epidemiology of 2019 novel coronavirus: implications for virus origins and receptor binding. Lancet 395: 565-574.

Mähler M., Berar M, Feinstein R., Gallagher A, Illgen-Wilcke B, Pritchett-Corning K, Raspa M. 2014. FELASA recommendations for the health monitoring of mouse, rat, hamster, guinea pig and rabbit colonies in breeding and experimental units. Lab. Anim. 48: 178-192.

Malik YA. 2020. Properties of coronavirus and SARS-CoV-2. Malay. J. Pathol. 42: 3-11.

McCray PB, Pewe L, Wohlford-Lenane C, Hickey M, Manzel L, Shi L, Netland J, Jia HP, Halabi C, Sigmund CD, Meyerholz DK, Kirby P, Look DC, Perlman S. 2007. Lethal Infection of K18hACE2 Mice Infected with Severe Acute Respiratory Syndrome Coronavirus. J. Virol. 81: 813-821.

Moreau GB, Burgess SL, Sturek JM, Donlan AN, Petri WA, Mann BJ. 2020. Evaluation of K18-hACE2 Mice as a Model of SARSCoV-2 Infection. Am. J. Trop. Med. Hyg. 103: 1215-1219.

Muñoz-Fontela C, Dowling WE, Funnell SGP, Gsell PS, RiverosBalta AX, Albrecht RA, Andersen $H$, Baric RS, Carroll MW, Cavaleri M, Qin C, Crozier I, Dallmeier K, de Waal L, de Wit E, Delang L, Dohm E, Duprex WP, Falzarano D, Barouch DH. 2020. Animal models for COVID-19. Nature 586: 509-515.

Nguyen HL, Lan PD, Thai NQ, Nissley DA, O'Brien EP, Li MS. 2020. Does SARS-CoV-2 bind to human ACE2 more strongly than does SARS-CoV? J. Phys. Chem. B. 124: 7336-7347.

Oladunni FS, Park JG, Pino PA, Gonzalez O, Akhter A, AlluéGuardia A, Olmo-Fontánez A, Gautam S, Garcia-Vilanova A, Ye C, Chiem K, Headley C, Dwivedi V, Parodi LM, Alfson KJ, Staples HM, Schami A, Garcia JI, Whigham A, Torrelles JB. 2020. Lethality of SARS-CoV-2 infection in K18 human angiotensinconverting enzyme 2 transgenic mice. Nat. Commun. 11: 1-17.

Organización Mundial de la Salud (OMS). 2021. Orientaciones sobre la bioseguridad en el laboratorio relacionada con la COVID-19. Orientaciones provisionales. https://apps.who.int/iris/handle/10665/339696 [Consulta 29 de julio de 2021]

Prasad A \& Prasad M. 2020. Single Virus Targeting Multiple Organs: What We know and Where We Are Heading? Front. Med. 7: 1-6.

Ren LL, Wang YM, Wu ZQ, Xiang ZC, Guo L, Xu T, Jiang YZ, Xiong Y, Li YJ, Li XW, Li H, Fan GH, Gu XY, Xiao Y, Gao H, Xu JY, Yang F, Wang XM, Wu C, Wang JW. 2020. Identification of a novel coronavirus causing severe pneumonia in human: a descriptive study. Chinese. Med. J-Peking. 133: 1015-1024. 
Rosa RB, Dantas WM, do Nascimento JCF, da Silva MV, de Oliveira RN, Pena LJ. 2021. In vitro and in vivo models for studying SARS-CoV-2, the etiological agent responsible for COVID-19 pandemic. Viruses 13: 1-29.

Su S, Wong G, Shi W, Liu J, Lai ACK, Zhou J, Liu W, Bi Y, Gao GF. 2016. Epidemiology, Genetic Recombination, and Pathogenesis of Coronaviruses. Trends. Microbiol. 24: 490-502.

Truett G, Heeger P, Mynatt R, Truett A, Walker J, Warman M. 2000. Preparation of PCR- Quality Mouse Genomic DNA with Hot Sodium Hydroxide and Tris (HotSHOT). BioTechniques 29: 52-54.

Tseng CTK, Huang C, Newman P, Wang N, Narayanan K, Watts DM, Makino S, Packard MM, Zaki SR, Chan T, Peters CJ. 2007. Severe Acute Respiratory Syndrome Coronavirus Infection of Mice Transgenic for the Human Angiotensin-Converting Enzyme 2 Virus Receptor. J. Virol. 81: 1162-1173.

Walls AC, Park YJ, Tortorici MA, Wall A, McGuire AT, Veesler D. 2020. Structure, Function, and Antigenicity of the SARS-CoV-2 Spike Glycoprotein. Cell 180: 281-292.

Wan Y, Shang J, Graham R, Baric RS, Li F. 2020. Receptor Recognition by the Novel Coronavirus from Wuhan: an Analysis Based on Decade-Long Structural Studies of SARS Coronavirus. J. Virol. doi:10.1128/JVI.00127-20.

Weiss SR, Leibowitz JL. 2011. Coronavirus pathogenesis. In Advances in Virus Research. Elsevier Inc. Pp.85-164. ISSN 00653527.

Winkler ES, Bailey AL, Kafai NM, Nair S, McCune BT, Yu J, Fox JM, Chen RE, Earnest JT, Keeler SP, Ritter JH, Kang LI, Dort S, Robichaud A, Head R, Holtzman MJ, Diamond MS. 2020. SARSCoV-2 infection of human ACE2-transgenic mice causes severe lung inflammation and impaired function. Nat. Immunol. 21: 1327-1335.

Wrapp D, Wang N, Corbett KS, Goldsmith JA, Hsieh CL, Abiona O, Graham BS, McLellan JS. 2020. Cryo-EM structure of the 2019-nCoV spike in the prefusion conformation. Science 367: 1260-1263.

Wu C, Chen X, Cai Y, Xia J, Zhou X., Xu S, Huang H, Zhang L, Zhou X, Du C, Zhang Y, Song J, Wang S, Chao Y, Yang Z, Xu J, Zhou X, Chen D, Xiong W, Song Y. 2020. Risk Factors Associated with Acute Respiratory Distress Syndrome and Death in Patients with Coronavirus Disease 2019 Pneumonia in Wuhan, China. JAMA Intern. Med. 180: 934-943.

Wu F, Zhao S, Yu B, Chen YM, Wang W, Song ZG, Hu Y, Tao ZW, Tian JH, Pei YY, Yuan ML, Zhang YL, Dai FH, Liu Y, Wang QM, Zheng JJ, Xu L, Holmes EC, Zhang YZ. 2020. A new coronavirus associated with human respiratory disease in China. Nature 579: 265-269.

Xia S, Wu M, Chen S, Zhang T, Ye L, Liu J, Li H. 2020. Long Term Culture of Human Kidney Proximal Tubule Epithelial Cells Maintains Lineage Functions and Serves as an Ex vivo Model for Coronavirus Associated Kidney Injury. Virol Sin. 35: 311320.

Xu X, Chen P, Wang J, Feng J, Zhou H, Li X, Zhong W, Hao P. 2020. Evolution of the novel coronavirus from the ongoing Wuhan outbreak and modeling of its spike protein for risk of human transmission. Sci. China. Life. Sci. 63: 457-460.
Yuan L, Tang Q, Cheng T, Xia N. 2020. Animal models for emerging coronavirus: progress and new insights. Emerg. Microbes. Infec. 9: 949-961.

Zaki AM, van Boheemen S, Bestebroer TM, Osterhaus ADME, Fouchier RAM. 2012. Isolation of a Novel Coronavirus from a Man with Pneumonia in Saudi Arabia. New. Engl. J. Med. 367: 1814-1820.

Zhang H, Penninger JM, Li Y, Zhong N, Slutsky AS. 2020. Angiotensin-converting enzyme 2 (ACE2) as a SARS-CoV-2 receptor: molecular mechanisms and potential therapeutic target. Intens. Care. Med. 46: 586-590.

Zheng J, Wong LYR, Li K, Verma AK, Ortiz ME, Wohlford-Lenane $C$, Leidinger MR, Knudson CM, Meyerholz DK, McCray PB, Perlman S. 2021. COVID-19 treatments and pathogenesis including anosmia in K18-hACE2 mice. Nature 589: 603-607.

Zheng J. 2020. SARS-CoV-2: An emerging coronavirus that causes a global threat. Int. J. Biol. Sci. 16: 1678-1685.

Zhou P, Yang XL, Wang XG, Hu B, Zhang L, Zhang W, Si HR, Zhu $Y$, Li B, Huang CL, Chen H D, Chen J, Luo Y, Guo H, Jiang RD, Liu MQ, Chen Y, Shen XR, Wang X, Shi ZL. 2020. A pneumonia outbreak associated with a new coronavirus of probable bat origin. Nature 579: 270-273.

Zhu N, Zhang D, Wang W, Li X, Yang B, Song J, Zhao X, Huang B, Shi W, Lu R, Niu P, Zhan F, Ma X, Wang D, Xu W, Wu G, Gao GF, Ta W. 2020. A Novel Coronavirus from Patients with Pneumonia in China, 2019. New. Engl. J. Med. 382: 727-733.

Zou X, Chen K, Zou J, Han P, Hao J, Han Z. 2020. Single-cell RNA-seq data analysis on the receptor ACE2 expression reveals the potential risk of different human organs vulnerable to 2019-nCoV infection. Front. Med-Prc. 14: 185-192. 